\title{
CHARACTERIZATIONS OF MINIMAL HAUSDORFF SPACES
}

\author{
JAMES E. JOSEPH
}

\begin{abstract}
Two new characterizations of minimal Hausdorff spaces are given along with some relating properties and examples.
\end{abstract}

1. Introduction. In a recent paper [4], Professors L. L. Herrington and P. E. Long used the notions of the $\boldsymbol{\theta}$-adherence and the convergence of a filterbase to characterize minimal Hausdorff spaces (see [1] for a survey of minimal topological spaces). In this paper, we employ their characterization to obtain characterizations of minimal Hausdorff spaces in terms of the graphs of functions into the spaces and a class $\mathcal{S}$ of spaces including the class of Hausdorff completely normal and fully normal spaces as a subclass.

2. Preliminaries. In this paper, we denote the closure of a subset $K$ of a space by $\operatorname{cl}[K]$. If $g, f: X \rightarrow Y$ are functions, we denote $\{x \in X: g(x)=f(x)\}$ by $\Omega(f, g, X, Y)$.

2.1. Definition [5]. A point $x$ is in the $\theta$-closure of a subset $K$ of a space $(x \in \theta$-cl $[K])$ if every open set $V$ about $x$ satisfies $K \cap \operatorname{cl}[V] \neq \varnothing$.

2.2. Definition [5]. A point $x$ is in the $\theta$-adherence of a filterbase $\mathcal{F}$ on a space $(x \in \theta-\operatorname{adh} \mathscr{F})$ if $x \in \theta-\operatorname{cl}[F]$ for each $F \in \mathscr{F}$. In this case, we will sometimes say that $x$ is a $\theta$-adherent point of $\mathscr{F}$.

2.3. Definition [3]. A function $g: X \rightarrow Y$ has a strongly-closed graph if for each $(x, y) \in(X \times Y)-G(g)$, there are open sets $V \subset X, W \subset Y$ about $x$ and $y$, respectively, satisfying $(V \times \operatorname{cl}[W]) \cap G(g)=\varnothing$.

2.4. Definition. Let $X$ be a set, let $x_{0} \in X$, and let $\mathcal{F}$ be a filterbase on $X$; $\left\{A \subset X: x_{0} \in X-A\right.$ or $F \cup\left\{x_{0}\right\} \subset A$ for some $\left.F \in \mathscr{F}\right\}$ is a topology on $X$ which will be called the topology on $X$ associated with $x_{0}$ and $\mathscr{F} . X$ equipped with this topology will be called the space $X$ associated with $x_{0}$ and $\mathscr{F}$. We will denote this space by $X\left(x_{0}, \mathscr{F}\right)$.

The following readily established theorem is used in the sequel.

2.5. Theorem. Let $X$ be a set, let $x_{0} \in X$ and let $\mathcal{F}$ be a filterbase on $X$ which has empty intersection on $X-\left\{x_{0}\right\}$. Then $X\left(x_{0}, \mathcal{F}\right)$ is in class $\mathcal{S}$.

3. Main results. We use the following characterization of minimal Hausdorff spaces.

Received by the editors February 26, 1976 and, in revised form, May 7, 1976.

AMS (MOS) subject classifications (1970). Primary 54D25, 54D30; Secondary 54C10.

Key words and phrases. Minimal Hausdorff spaces, functions with closed graphs. 
3.1. Definition [4, p. 421]. A Hausdorff space is minimal Hausdorff if each filterbase on the space possessing at most one $\theta$-adherent point is convergent.

The following result [4, Theorem 7] will also be used.

3.1. Theorem. Let $f: X \rightarrow Y$ be a function and let $Y$ be minimal Hausdorff. If $f$ has a strongly-closed graph, then $f$ is continuous.

We turn now to our main results.

3.2. Theorem. A Hausdorff space $Y$ is minimal Hausdorff if and only if for each space $X$ in class $\mathcal{S}$ and bijections $f, g: X \rightarrow Y$ with closed graph and strongly-closed graph, respectively, $\Omega(f, g, X, Y)$ is closed in $X$.

Proof. Strong necessity. Let $Y$ be a minimal Hausdorff space, let $X$ be any space and let $f, g: X \rightarrow Y$ be any functions with closed graph and stronglyclosed graph, respectively. Since $g$ has a strongly-closed graph, it follows from Theorem 3.1 that $g$ is continuous and, consequently, that the restriction of the projection, $\pi_{x}: X \times Y \rightarrow X$, to $G(g)$ is a homeomorphism. $G(g) \cap G(f)$ is a closed subset of $G(g)$ since $f$ has a closed graph. Thus, $\Omega(f, g, X, Y)=$ $\pi_{x}(G(f) \cap G(g))$ is closed in $X$.

Sufficiency. Let $\mathcal{F}$ be a filterbase on the Hausdorff space $Y$ with at most one $\theta$-adherent point, $x_{0}$, in $Y$. If $\mathscr{F}$ does not converge to $x_{0}$, there is a $V_{0}$ open about $x_{0}$ such that $\mathscr{F}^{*}=\left\{F \cap\left(Y-V_{0}\right): F \in \mathscr{F}\right\}$ is a filterbase on $Y$. $Y\left(x_{0}, \mathscr{F}^{*}\right)$ is in class $\mathcal{S}$ from Theorem 2.1. Let $i: Y\left(x_{0}, \mathscr{F}^{*}\right) \rightarrow Y$ be the identity function; choose $y_{0} \in Y-\left\{x_{0}\right\}$ and define $g: Y\left(x_{0}, \mathscr{F}^{*}\right) \rightarrow Y$ by $g\left(x_{0}\right)=y_{0}, g\left(y_{0}\right)=x_{0}$, and $g(x)=x$ otherwise. Clearly, $i$ and $g$ are bijections; we show that $i$ has a strongly-closed graph, that $g$ has a closed graph, and that $\Omega\left(i, g, Y\left(x_{0}, \mathcal{F}^{*}\right), Y\right)$ is not closed in $Y\left(x_{0}, \mathscr{F}^{*}\right)$;

(a) $i$ has a strongly-closed graph. Let $x, y \in Y$ and $x \neq y$. If $x \neq x_{0}$, choose $W$ open in $Y$ about $y$ such that $x \notin \operatorname{cl}[W]$. Then $(\{x\} \times \operatorname{cl}[W]) \cap G(i)=\varnothing$ and $\{x\}$ is open in $Y\left(x_{0}, \mathscr{F}^{*}\right)$. If $x=x_{0}$, then $y \neq x_{0}$; so, since $x_{0}$ is the only possible $\theta$-adherent point for $\mathscr{F}$, there is an open set $W$ about $y$ and an $F \in \mathcal{F}$ satisfying $\left(F \cup\left\{x_{0}\right\}\right) \cap \operatorname{cl}[W]=\varnothing$. This implies that $\left(\left(F \cup\left\{x_{0}\right\}\right) \times\right.$ $\operatorname{cl}[W]) \cap G(i)=\varnothing$ and, since $F \cup\left\{x_{0}\right\}$ is open in $Y\left(x_{0}, \mathscr{F}^{*}\right)$, this completes the demonstration that $i$ has a strongly-closed graph;

(b) $g$ has a closed graph. Let $(x, y) \in\left(Y\left(x_{0}, \mathscr{F}^{*}\right) \times Y\right)-G(g)$. If $x \neq x_{0}$, choose $W$ open about $y$ satisfying $g(x) \notin W$. Then $\{x\}$ is open in $Y\left(x_{0}, \mathscr{F}^{*}\right)$ and $(\{x\} \times W) \cap G(g)=\varnothing$. Suppose $x=x_{0}$. Then $y \neq y_{0}$. If $y=x_{0}$, let $W$ be open in $Y$ about $y$, and $F \in \mathscr{F}$ such that $y_{0} \notin W \cup F$. This is possible since $Y$ is Hausdorff and $y_{0}$ is not an adherent point of $\mathscr{F}$.

$$
\left[\left(\left(F \cap\left(X-V_{0}\right)\right) \cup\left\{x_{0}\right\}\right) \times\left(V_{0} \cap W\right)\right] \cap G(g)=\varnothing
$$

and $\left(F \cap\left(X-V_{0}\right)\right) \cup\left\{x_{0}\right\}$ is open in $Y\left(x_{0}, F^{*}\right)$. If $y \neq x_{0}$, there is a $W$ open in $Y$ about $y$ and an $F \in \mathscr{F}$ satisfying $x_{0} \notin W, y_{0} \notin W$ and $F \cap W=$ $\varnothing$. $F \cup\left\{x_{0}\right\}$ is open in $Y\left(x_{0}, \mathscr{F}^{*}\right)$ and $\left(\left(F \cup\left\{x_{0}\right\}\right) \times W\right) \cap G(g)=\varnothing$. Thus, $g$ has a closed graph. 
The proof is complete since $\Omega\left(i, g, Y\left(x_{0}, \mathscr{F}^{*}\right), Y\right)=Y-\left\{x_{0}, y_{0}\right\}$ which is not closed in $Y\left(x_{0}, \mathscr{F}^{*}\right)$ because $x_{0} \in \operatorname{cl}\left[Y-\left\{x_{0}, y_{0}\right\}\right]$.

3.3. Theorem. A Hausdorff space $Y$ is minimal Hausdorff if and only if for each space $X$ in class $\mathcal{S}$ and functions $f, g: X \rightarrow Y$ with $f$ a bijection with a strongly-closed graph and $g$ a function with a closed graph, $\Omega(f, g, X, Y)=X$ whenever $\Omega(f, g, X, Y)$ is dense in $X$.

Proof. Strong necessity. Let $Y$ be minimal Hausdorff, let $X$ be any space and let $f, g: X \rightarrow Y$ be any functions with closed graph and strongly-closed graph, respectively; if $\Omega(f, g, X, Y)$ is dense in $X$, then $\Omega(f, g, X, Y)=X$ since $\Omega(f, g, X, Y)$ is closed in $X$ from the proof of the strong necessity of Theorem 3.2.

Sufjiciency. We follow the proof of the sufficiency of Theorem 3.2 to the point immediately preceding the definition of $g$. We choose $y_{0} \in X-\left\{x_{0}\right\}$ and define $g: Y\left(x_{0}, \mathscr{F}^{*}\right) \rightarrow Y$ by $g(x)=x$ if $x \neq x_{0}$ and $g\left(x_{0}\right)=y_{0}$. By arguments similar to those in the proof of the sufficiency of Theorem 3.2, we show that $g$ has a closed graph and that the identity function $i$ has a strongly-closed graph. $\Omega\left(i, g, Y\left(x_{0}, F^{*}\right), Y\right)=Y-\left\{x_{0}\right\}$ which is dense in $Y\left(x_{0}, \mathscr{F}^{*}\right)$. This is a contradiction which completes the proof.

4. Some examples. In this section, we give some examples to indicate some of the limitations on the weakening of hypotheses in the theorems in this paper. By way of notation, we let $N$ be the set of positive integers and for each $k \in N$, we let $O(k)=\{k+1 / 2 n: n \in N\}, E(k)=\{k+1 /(2 n-1)$ : $n \in N\}, Z(k)=\{n \in N: n \geqslant k\}$; we let $Y=\{-1,0\} \cup \cup_{N} O(k) \cup$ $\cup_{N} E(k) \cup N$. Let $X=\{0\} \cup \cup_{N} E(k) \cup N$.

4.1. EXAMPLE. The requirement cannot be weakened to all functions having closed graphs in either of Theorems 3.1,3.2, or 3.3. Let $Y$ have the topology generated by the collection of all sets in the three collections, $\{\{-1\} \cup$ $\left.\cup_{k \geqslant m} O(k): m \in N\right\}, \quad\left\{\{0\} \cup \cup_{k \geqslant m} E(k): m \in N\right\}$, and $\{V \cap(Y-$ $\{-1,0\}): V$ is a usual open subset of the reals $\}$ as base. $Y$ with this topology is known to be minimal Hausdorff [2]. We let $\mathscr{F}=\{Z(k): k \in N\}$. Let $i$ : $Y(1, \mathscr{F}) \rightarrow Y$ be the identity function; we define $g: Y(1, \mathscr{F}) \rightarrow Y$ by $g(0)=1$, $g(1)=0$ and $g(x)=x$ otherwise. We define $h: Y(1, \mathcal{F}) \rightarrow Y$ by $h(1)=0$ and $h(x)=x$ otherwise. $i, g$, and $h$ all have closed graphs which are not strongly-closed. $i$ is not continuous at $x=1$.

$\Omega(i, g, Y(1, \mathscr{F}), Y)=Y-\{0,1\}$ which is not closed in $Y(1, \mathscr{F})$.

$\Omega(i, h, Y(1, \mathscr{F}), Y)=Y-\{1\}$ which is dense in $Y(1, \mathscr{F})$.

4.2. EXAMPLE. The requirement cannot be changed to all functions having strongly-closed graphs in either of Theorems 3.2 or 3.3. Let $X$ have the relative topology from $Y . X$ is known to be $H$-closed and is not minimal Hausdorff [1]. Let $T$ be any space and let $g, f: T \rightarrow X$ have strongly-closed graphs. We will show that $\Omega(g, f, T, X)$ is closed in $T$. We remark that by Theorem 9 in [3], for each $v \in T$ and $W$ open in $X$ about $g(v)$, there is a $Q$ open in $T$ about $v$ satisfying $g(Q) \subset \operatorname{cl}[W]$. Let $v \in \operatorname{cl}[\Omega(f, g, T, X)]$ and suppose $f(v) \neq g(v)$. 
There is a $V$ open in $T$ about $v$ and a $W$ open in $X$ about $g(v)$ satisfying $(V \times \operatorname{cl}[W]) \cap G(f)=\varnothing$. There is an $A$ open in $T$ about $v$ satisfying $g(A) \subset \mathrm{cl}[W]$. Then $A \cap V$ is open in $T, v \in A \cap V$, and $(A \cap V) \cap$ $\Omega(f, g, T, X)=\varnothing$. This contradiction shows that $\Omega(f, g, T, X)$ is closed.

\section{REFERENCES}

1. M. P. Berri, J. R. Porter and R. M. Stephenson, Jr., A survey of minimal topological spaces, General Topology and its Relations to Modern Analysis and Algebra, III (Proc. Conf., Kanpur, 1968), Academia, Prague, 1971, pp. 93-114. MR 43 \#3985.

2. N. Bourbaki, Espaces Minimaux et espaces complètement séparés, C. R. Acad. Sci. Paris 212 (1941), 215-218. MR 3, 136.

3. L. L. Herrington and P. E. Long, Characterizations of $H$-closed spaces, Proc. Amer. Math. Soc. 48 (1975), 469-475. MR 51 \# 1737.

4. Characterizations of C-compact spaces, Proc. Amer. Math. Soc. 52 (1975), $417-426$.

5. N. V. Veličko, H-closed topological spaces, Mat. Sb. 70(112) (1966), 98-112; English transl., Amer. Math. Soc. Transl. (2) 78 (1969), 103-118. MR 33 \#6576.

Department of Mathematics, Federal City College, Washington, D.C. 20001 